POS PROCEEDINGS

\title{
High Mass Higgs boson Searches in ATLAS and CMS
}

\author{
Joana MACHADO MIGUENS* ${ }^{*}$, on behalf of the ATLAS and CMS Collaborations \\ Faculdade de Ciencias, Universidade de Lisboa and LIP, Lisboa, Portugal \\ E-mail: jmiguens@cern.ch
}

\begin{abstract}
The recently discovered resonance at $125 \mathrm{GeV}$ shows properties, so far, consistent with the Standard Model Higgs boson. It is, nonetheless, important to directly search the full mass range available at the Large Hadron Collider (LHC). Searches for a high-mass Higgs boson, with SM-like properties, can be performed without placing any bias on the nature of the new particle. Moreover, they can be used as a starting point for Beyond the Standard Model scenarios, that can be studied by simply rescaling the Standard Model analyses. The latest results from ATLAS and CMS, using data from the LHC to search for SM-like Higgs bosons with masses substantially above the observed $125 \mathrm{GeV}$ Higgs boson, are presented. These include $Z Z$ and $W W$ decay modes and explore the $200 \mathrm{GeV}$ to $1 \mathrm{TeV}$ mass range, where no significant excesses have been observed.
\end{abstract}

The European Physical Society Conference on High Energy Physics

18-24 July, 2013

Stockholm, Sweden

\footnotetext{
* Speaker.

${ }^{\dagger}$ Work financed in part by FCT, Portugal, through the grant SFRH/BD/69173/2010, co-financed by GRICES and FCT, Portugal.
} 


\section{Introduction}

ATLAS [1] and CMS [2] are two general-purpose detectors at the Large Hadron Collider (CERN), that have successfully collected more than $25 \mathrm{fb}^{-1}$ of proton-proton collision data. The ATLAS and CMS Collaborations have recently announced the discovery of a new particle [3, 4], with a mass of approximately $125 \mathrm{GeV}$ and properties [5, 6], so far, consistent with the Standard Model (SM) Higgs boson. The question remains, however, if this particle is fully responsible for electroweak (EW) symmetry breaking, or if additional beyond the SM (BSM) physics, predicting more than one Higgs boson, exists to play this role. It is, therefore, crucial to explore the full accessible mass range, up to $1 \mathrm{TeV}$, for which the $H \rightarrow W W$ and $H \rightarrow Z Z$ decay modes are favoured.

The simplest extension of the SM Higgs sector predicts a heavy real EW singlet [7] mixing with the $H(125)$ particle. In this model, both gauge and fermion couplings of the heavy state $(H(125))$ are scaled with respect to the SM, by a common factor $\kappa^{\prime}(\kappa)$, with the relation $\kappa^{2}+\kappa^{\prime 2}=1$ imposed by unitarity. The heavy state can have "non-SM-like" decays, including the decay to $H(125)$, which are parametrized by $\mathrm{BR}_{\text {new }}$, such that $\mathrm{BR}^{\prime}=\left(1-\mathrm{BR}_{\text {new }}\right) \mathrm{BR}_{\mathrm{SM}}$. Current measurements on the $H(125)$ favour a heavy state with a narrow width [7], which is modelled using the Narrow Width Approximation (NWA). To model a heavy resonance with a larger, SM-like width, analyses currently use the Complex Pole Scheme (CPS), since the Breit-Wigner description of the lineshape is not valid for $m_{H}>400 \mathrm{GeV}^{*}$.

This paper presents the most recent efforts by ATLAS and CMS in the search for high-mass Higgs bosons, exploring the following decay modes: $H \rightarrow W W \rightarrow \ell v q q, H \rightarrow W W \rightarrow \ell v \ell v, H \rightarrow$ $Z Z \rightarrow \ell \ell \ell \ell, H \rightarrow Z Z \rightarrow \ell \ell v v$ and $H \rightarrow Z Z \rightarrow \ell \ell q q$.

\section{2. $H \rightarrow W W \rightarrow \ell v q q$}

Analyses in the $\ell v q q$ final state search for one high transverse momentum $\left(p_{\mathrm{T}}\right)$, isolated lepton, either an electron $(e)$ or a muon $(\mu)$, large missing transverse energy $\left(E_{\mathrm{T}}^{\mathrm{miss}}\right)$ and jet(s) compatible with a $W$ decay to quarks. The full invariant mass of the system, used to extract the signal, can be reconstructed by placing the constraint $m_{\ell v}=m_{W} . W+$ jets events are the dominant background.

ATLAS has performed a search in the $H \rightarrow W W \rightarrow \ell v q q$ channel [8], using $4.7 \mathrm{fb}^{-1}$ of $\sqrt{s}=$ $7 \mathrm{TeV}$ data and exploring the $300-600 \mathrm{GeV}$ mass range. The signal is extracted by fitting the $m_{\ell v j j}$ spectrum in data, with a functional form tested in the $m_{j j}$ sidebands. No significant excesses of data above the background model were observed and upper limits on $\sigma / \sigma_{\mathrm{SM}}$ (production cross-section normalized to the SM expectation) were extracted. The best sensitivity is reached at $m_{H}=400 \mathrm{GeV}$, where the 95\% CL (confidence level) observed (expected) limits are 1.9 (1.6) times the SM for the categories with 0 and 1 additional jets, and $7.9(6.5)$ times the SM for events with 2 additional jets.

CMS's analysis in the $\ell v q q$ final state [9] uses $19.3 \mathrm{fb}^{-1}$ of $\sqrt{s}=8 \mathrm{TeV}$ data to exploit the $600-1000 \mathrm{GeV}$ mass range. Boosted $W$ bosons $\left(p_{\mathrm{T}}>200 \mathrm{GeV}\right)$ are selected, in which the decay quarks form a single "fat jet" $(J)$, reconstructed with a $\Delta R=0.8$ Cambridge-Aachen algorithm. The jet mass is pruned, to suppress multijet and pile-up contributions to the jet, and $\mathrm{N}$-subjettiness,

*Before the CPS prescription was available, conservative uncertainties, of the form $1.5\left(m_{H} / \mathrm{TeV}\right)^{3}$, were assigned to the production cross-section to cover for this lineshape effect, as well as for the signal-continuum background interference effect, that increases with the increasing width of the particle. 
an observable measuring the number of subjets in the jet, is used to further discriminate the hadronically decaying $W$ boson from multijet background. Both normalization and $m_{\ell v J}$ shape of $W+$ jets background in the final signal region, shown in Figure 1-middle, are extracted from fits to data in the $m_{J}$ sidebands, shown in Figure 1-left. No significant excesses were observed and 95\% CL upper limits on $\sigma / \sigma_{\mathrm{SM}}$ were set. Observed (expected) limits range from 1.1 (1.05) times the SM at $m_{H}=600 \mathrm{GeV}$, to 4.2 (4.6) times the SM at $1 \mathrm{TeV}$. In the BSM heavy EW singlet scenario, 95\% CL exclusion limits on $\sigma \times \mathrm{BR}$ of $H \rightarrow W W \rightarrow \ell v q q$ (cross section times branching ratio) are set for different $\kappa^{\prime}$ hypothesis $\left(C^{\prime}\right.$, used in figures from CMS, is equivalent to $\left.\kappa^{\prime}\right)$, assuming $\mathrm{BR}_{\text {new }}=0$. They range from $60 \mathrm{fb}$ to $400 \mathrm{fb}$ and are shown in Figure 1-right.
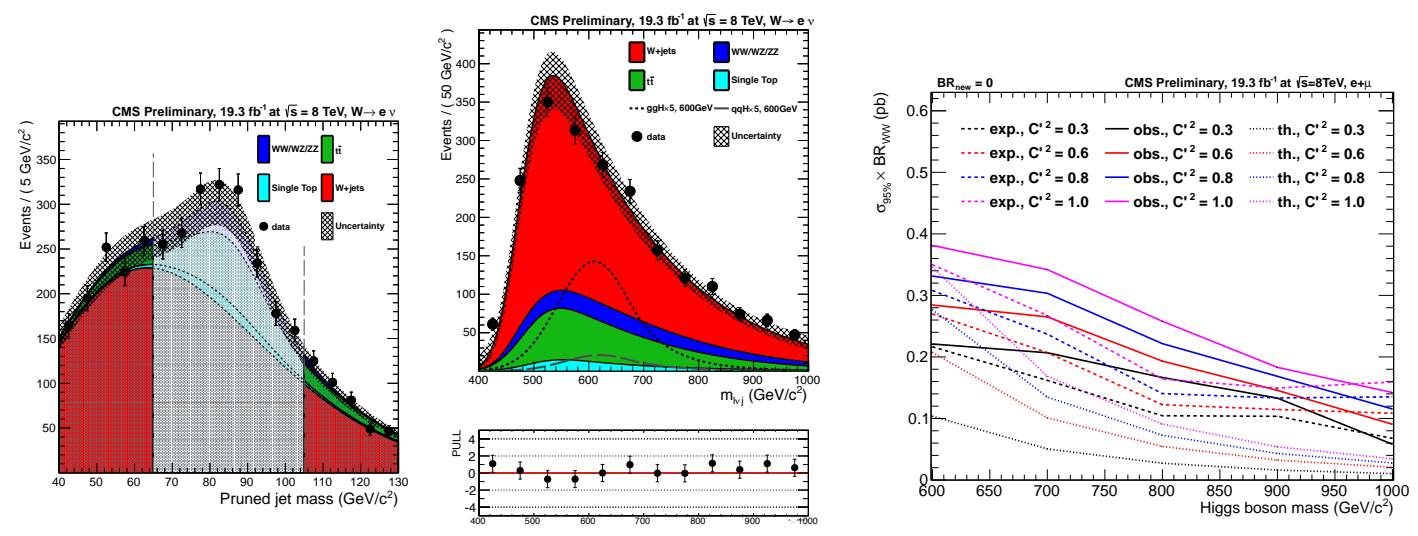

Figure 1: Left: Data and MC distribution for the $m_{J}$ sidebands and signal region. Middle: $m_{\ell v J}$ distribution in the signal region for electron events. Right: BSM exclusion limits as a function of mass for various values of $\kappa^{\prime 2}$, with $\mathrm{BR}_{\text {new }}=0$. Figures from Ref. [9].

\section{3. $H \rightarrow W W \rightarrow \ell v \ell v$}

The $H \rightarrow W W \rightarrow \ell v \ell v$ analysis is challenging, as the presence of two neutrinos in the final state does not allow for a full mass reconstruction. The basic signature of this channel consists of two high $p_{\mathrm{T}}$, isolated, opposite charge leptons, either electrons or muons, and large $E_{\mathrm{T}}^{\text {miss }}$. Continuum $W W$ production is the largest source of background.

ATLAS has analyzed the $\ell v \ell v$ channel [10] in the $260-1000 \mathrm{GeV}$ range, using $20.7 \mathrm{fb}^{-1}$ of $8 \mathrm{TeV}$ data. Events with 0,1 and 2 jets are used and SM-like and NWA hypothesis are investigated. High invariant mass of the dilepton system $\left(m_{\ell \ell}>50 \mathrm{GeV}\right)$ and and small pseudo-rapidity separation between the leptons $(\Delta \eta<1.0)$ are required to suppress the $H(125)$ resonance (considered background) and $W W$ background. Top and $W W$ processes are normalized using data control regions. The transverse mass, $m_{\mathrm{T}}=\sqrt{\left(E_{\mathrm{T}}^{\ell \ell}+E_{\mathrm{T}}^{\text {miss }}\right)^{2}-\left|\vec{p}_{\mathrm{T}}^{\ell \ell}+\vec{p}_{\mathrm{T}}^{\text {miss }}\right|^{2}}$, shown in Figure 2-left, is fitted to extract the signal. Data agrees with the background expectation and 95\% CL limits are set on $\sigma \times \mathrm{BR}$ of $H \rightarrow W W \rightarrow \ell v \ell v$, separately for gluon fusion (ggF) and vector boson fusion (VBF) production. Figure 2-middle shows these limits, as a function of $m_{H}$, for $g g F$ and NWA. Figure 2right shows the analysis excludes a SM-like Higgs in the range $260-642 \mathrm{GeV}$. At $m_{H}=600 \mathrm{GeV}$, upper limits on ggF (VBF) are 34 (16) fb for a SM-like width, and 32 (12) fb for NWA.

CMS has looked for a SM-like Higgs boson in the $H \rightarrow W W \rightarrow \ell v \ell v$ channel [11], using the full $7+8 \mathrm{TeV}$ datasets, searching in the $110-600 \mathrm{GeV}$ mass range. A powerful shape analysis, 

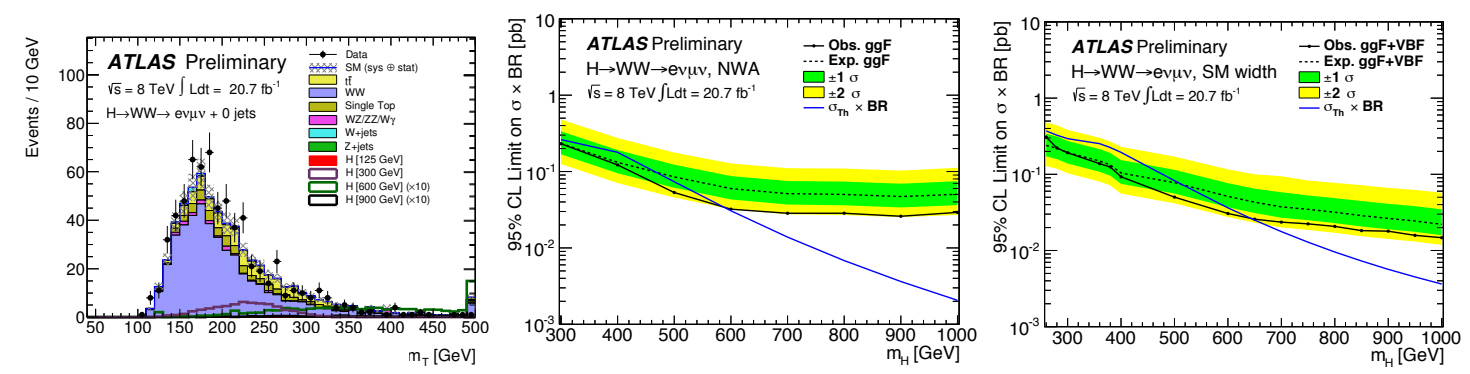

Figure 2: Left: Transverse mass distribution for events with no accompanying jets in the final signal region. Middle (Right): $95 \%$ CL upper limits on $\sigma \times \mathrm{BR}$ for $H \rightarrow W W \rightarrow \ell v \ell v$, for $g g F(\mathrm{ggF}+\mathrm{VBF}$ ) production of a Higgs with narrow (SM-like) width; the blue line represents the SM expectation. Figures from Ref. [10].

consisting of a two-dimensional (2D) fit of the data in $m_{\mathrm{T}}=\sqrt{2 p_{\mathrm{T}}^{\ell \ell} E_{\mathrm{T}}^{\text {miss }}\left(1-\cos \Delta \phi\left(E_{\mathrm{T}}^{\text {miss }}, \ell \ell\right)\right)}$ and $m_{\ell \ell}$, is used to constrain the backgrounds and extract the signal in $e \mu$ final states. A 2D histogram of $m_{\ell \ell}$ versus $m_{\mathrm{T}}$ is shown in Figure 3-left, for data minus expected background. For events with same flavour leptons in the final state, a cut-based approach is used. Upper limits on $\sigma / \sigma_{\mathrm{SM}}$ are placed and a Higgs boson with a mass in the range $128-600 \mathrm{GeV}$ is excluded at $95 \% \mathrm{CL}$ (for an expectation of $115-575 \mathrm{GeV})$. As shown in Figure 3-right, no significant excesses are observed over the expectation, once the $H(125)$ resonance is included as background process.
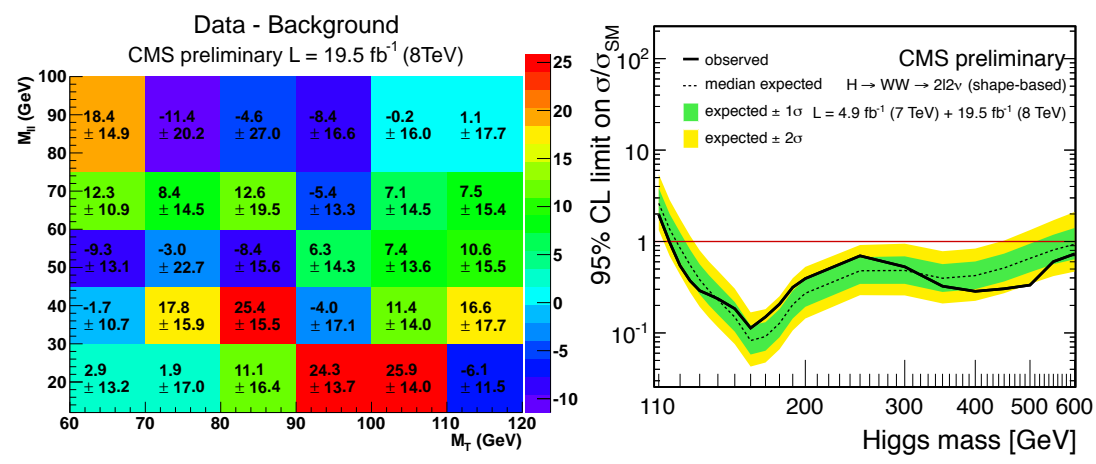

Figure 3: Left: 2D $m_{\mathrm{T}}-m_{\ell \ell}$ distribution for data minus background in events with no jets. Right: 95\% CL upper limits on $\sigma / \sigma_{\mathrm{SM}}$, where a Higgs boson with $m_{H}=125 \mathrm{GeV}$ has been added to the background processes. Figures from Ref. [11].

\section{4. $H \rightarrow Z Z \rightarrow \ell \ell \ell \ell$}

Even though the branching fraction is low, the $H \rightarrow Z Z \rightarrow \ell \ell \ell \ell$ channel profits from very high signal-to-background ratio and a very clean signature, consisting of two pairs of same flavour, opposite charge, isolated, high- $p_{\mathrm{T}}$ leptons. Moreover, the full $m_{4 \ell}$ invariant mass can be reconstructed with excellent resolution. Continuum $Z Z$ production is the dominant background in the analyses.

The ATLAS analysis in the 4-lepton channel [12] considers final states with electrons and/or muons and splits events according to flavour. Further categorization, targeting different production modes, is used: the VBF-like category includes events with two high- $p_{\mathrm{T}}$ jets with a large rapidity gap; the associated production $(\mathrm{VH})$ category requires an additional lepton; and the ggF-like category includes the remaining events. The $m_{4 \ell}$ distribution is used as a signal discriminant and shows no significant excesses. 95\% CL limits on $\sigma \times \mathrm{BR}$ for $H \rightarrow Z Z \rightarrow 4 \ell$ are estimated separately for ggF, shown in Figure 4-left, and combined VBF/VH production modes, assuming a SM-like Higgs.

CMS has analyzed the $H \rightarrow Z Z \rightarrow \ell \ell \ell \ell$ channel [13], considering not only electron and muon final states, but also hadronic and leptonic $\tau$ decays for one of the $Z$ bosons, which increases the 
sensitivity at high mass. For this category, the reconstructed visible mass $m_{2 \ell 2 \tau}$, shown in Figure 4middle, is used to discriminate signal from background. For the categories with only electrons or muons, different signal discriminants are used, depending on the number of accompanying jets. $m_{4 \ell}, k_{D}$, a likelihood ratio kinematic discriminant built from a matrix element likelihood approach using decay and production angles, and $p_{\mathrm{T}} / m_{4 \ell}$ (for $m_{H}<160 \mathrm{GeV}$ ) are used for events with fewer than two jets. $m_{4 \ell}, k_{D}$ and $V_{D}$, a linear discriminant using VBF-sensitive variables, are used for events with two or more jets. The observed distributions agree well with the background expectation. Upper limits on $\sigma / \sigma_{\mathrm{SM}}$ are calculated using all channels, as shown in Figure 4-right. The analysis excludes a SM-like Higgs boson at $95 \% \mathrm{CL}$ in the range $130-827 \mathrm{GeV}$, for an expectation of $113.5-778 \mathrm{GeV}$.
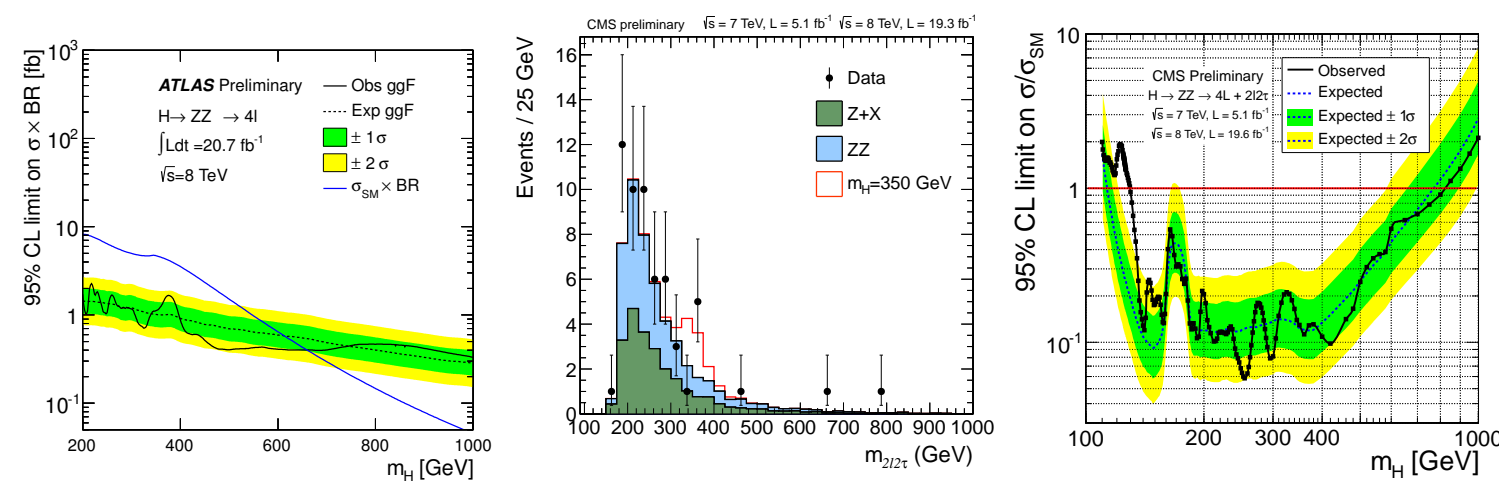

Figure 4: Left: $95 \% \mathrm{CL}$ upper limit on $\sigma \times \mathrm{BR}$ of $H \rightarrow Z Z \rightarrow \ell \ell \ell \ell$ for a ggF produced SM-like signal as a function of $m_{H}$; Figure from Ref. [12]. Middle: Distribution of the four-lepton reconstructed mass for channels with one $\tau$ decay. Right: Observed and expected 95\% CL limits on $\sigma / \sigma_{\mathrm{SM}}$. Figures from Ref. [13].

\section{5. $H \rightarrow Z Z \rightarrow \ell \ell v v$}

The branching fraction for the $H \rightarrow Z Z \rightarrow \ell \ell v v$ channel is high, but no full mass reconstruction is possible. Analyses require two high- $p_{\mathrm{T}}$, isolated leptons, either electrons or muons, compatible with a $Z$ boson decay, and very large $E_{\mathrm{T}}^{\text {miss }}$. Inclusive $Z, Z Z$, top and other diboson processes contribute as backgrounds.

ATLAS has searched the $200-600 \mathrm{GeV}$ mass range in this channel [14] using $4.7 \mathrm{fb}^{-1}$ of $7 \mathrm{TeV}$ data. The transverse mass, $m_{\mathrm{T}}^{2}=\left[\sqrt{m_{Z}^{2}+\left|\vec{p}_{\mathrm{T}}^{\ell \ell}\right|^{2}}+\sqrt{m_{Z}^{2}+\left|\vec{p}_{\mathrm{T}}^{\text {miss }}\right|^{2}}\right]^{2}-\left[\vec{p}_{\mathrm{T}}^{\ell \ell}+\vec{p}_{\mathrm{T}}^{\text {miss }}\right]^{2}$, distribution was used to extract the signal. Since no significant excess was observed, upper limits on $\sigma / \sigma_{\mathrm{SM}}$ were set. A SM-like Higgs boson is excluded at $95 \% \mathrm{CL}$ in the $319-558 \mathrm{GeV}$ mass range (for an expectation of $280-497 \mathrm{GeV}$ ).

CMS has performed a search in the $\ell \ell v v$ final state [15], using the full $7+8 \mathrm{TeV}$ datasets and analyzing the $200-1000 \mathrm{GeV}$ mass range. Events are categorized according to lepton flavour and sensitivity to ggF or VBF production mode. $Z+$ jets background events are modelled using $\gamma+$ jet events in data. Non-resononant background processes are normalized from events with different flavour leptons in the $m_{\ell \ell}$ sidebands of the $Z$. Optimized $E_{\mathrm{T}}^{\text {miss }}$ and $m_{\mathrm{T}}$ (similar definition to ATLAS, with $m_{Z}$ replaced by $m_{\ell \ell}$ ) selections are applied depending on the category. The signal is extracted by fitting the $m_{\mathrm{T}}$ distribution for the $\mathrm{ggF}$ categories and the $E_{\mathrm{T}}^{\mathrm{miss}}$ distribution for the VBF category. 
No excess of events above the expectation was observed and upper limits on $\sigma / \sigma_{\mathrm{SM}}$ were set, excluding a SM-like Higgs boson at $95 \% \mathrm{CL}$ in the $248-930 \mathrm{GeV}$ mass range (for an expectation of $254-898 \mathrm{GeV}$ ), as shown in Fig 5-left. Results were also interpreted in the BSM heavy EW singlet scenario. Fig 5-middle shows limits on $\sigma \times \mathrm{BR}$ for $H \rightarrow Z Z \rightarrow \ell \ell v v$ as a function of $m_{H}$, considering $\mathrm{ggF}$ production, $\mathrm{BR}_{\text {new }}=0$ and different hypothesis on $\kappa^{\prime}$. The observed lines fall below the theory lines for large $\kappa^{\prime}$ hypothesis, excluding the heavy EW singlet in a large mass range. Similar upper limits, with different hypotheses on both $\kappa^{\prime}$ and $\mathrm{BR}_{\text {new }}$, are shown in Figure 5right, for $m_{H}=400 \mathrm{GeV}$. The hatched area indicates the excluded phase-space, with a narrow-width boson being favoured.
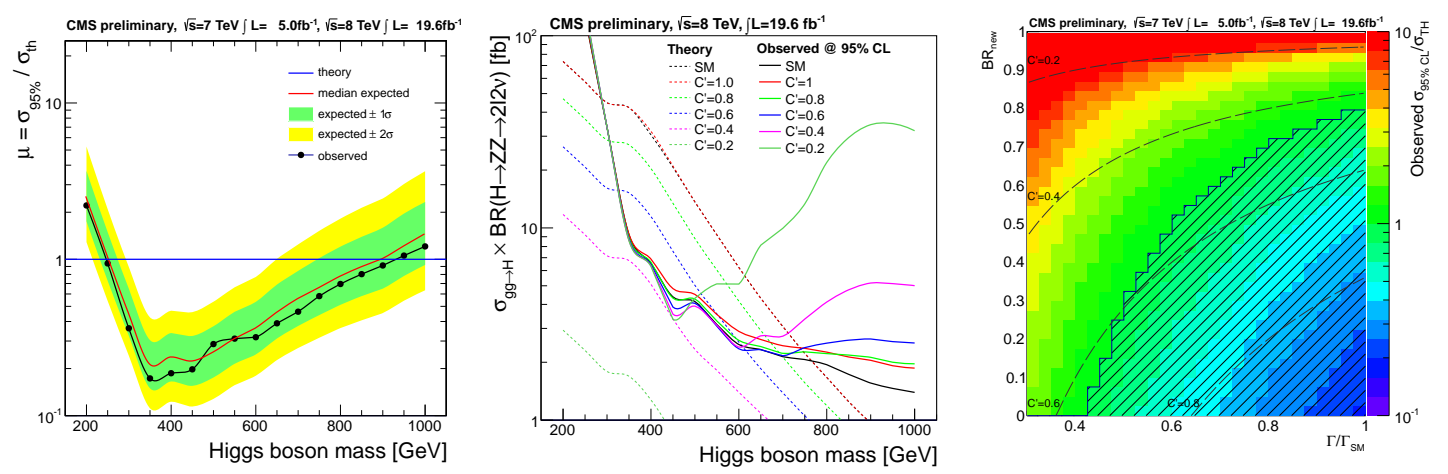

Figure 5: Left: Expected and observed 95\% CL limits on $\sigma / \sigma_{\mathrm{SM}}$ as a function of $m_{H}$. Middle: Observed 95\% CL upper limits on $\sigma \times \mathrm{BR}$ for $\mathrm{ggF} H \rightarrow Z Z \rightarrow \ell \ell v v$, as function of $m_{H}$, for different $\kappa^{\prime}$ hypothesis and under the assumption $\mathrm{BR}_{\text {new }}=0$. Right: Observed upper limits at $95 \% \mathrm{CL}$ on $\sigma$, normalized to the theory cross-section assuming SM contributions from $\mathrm{ggF}$ and $\mathrm{VBF}$, for $m_{H}=400 \mathrm{GeV}$, as function of both $\kappa^{\prime}$ and the width of the EW singlet relative to the SM, which follows the relation $\Gamma^{\prime} / \Gamma_{\mathrm{SM}}=\kappa^{\prime 2} /\left(1-\mathrm{BR}_{\text {new }}\right)$; the hatched area represents the excluded region. Figures from Ref. [15].

6. $H \rightarrow Z Z \rightarrow \ell \ell q q$

Analyses in the $H \rightarrow Z Z \rightarrow \ell \ell q q$ channel require two high- $p_{\mathrm{T}}$, isolated leptons, either electrons or muons, compatible with a $Z$-boson decay, and two jets, also compatible with $Z$ decays. Jets are more often $b$-jets for signal than for $Z+$ jets, which constitutes the largest background, a feature that is exploited to discriminate signal and background. The full $m_{\ell \ell j j}$ mass can be reconstructed and is used to extract the signal.

ATLAS has analyzed the $\ell \ell j j$ final state [16], in the $200-600 \mathrm{GeV}$ mass range, using $4.7 \mathrm{fb}^{-1}$ of $7 \mathrm{TeV}$ data. The normalization and flavour composition of the $Z+$ jets background are derived directly from data using the $m_{j j}$ sidebands and the $b$-tagging discriminant, whereas the shapes are extracted from simulation with small corrections from data. No significant excesses were observed and $95 \% \mathrm{CL}$ upper limits on $\sigma / \sigma_{\mathrm{SM}}$ were set. The analysis excludes a SM-like Higgs boson in the $300-322 \mathrm{GeV}$ and $353-410 \mathrm{GeV}$ mass ranges, for an expectation of $351-404 \mathrm{GeV}$.

The analysis performed by CMS in the $H \rightarrow Z Z \rightarrow \ell \ell q q$ channel [17] uses the full $7+8 \mathrm{TeV}$ datasets to search the $230-600 \mathrm{GeV}$ mass range. An angular likelihood discriminant is built from production and decay angles, and used to separate signal from background. Events are categorized according to $b$-tagging and a $E_{\mathrm{T}}^{\text {miss }}$-based selection is used to suppress $t \bar{t}$ background. The $Z+$ jets normalization is derived using data in the $m_{j j}$ sidebands and the shape is based on simulation with 
data corrections. Figure 6-left shows the invariant mass, $m_{\ell \ell j j}$, distribution, which is fitted to extract the signal. No significant excesses of data over the expected background are observed and upper limits on $\sigma / \sigma_{S M}$ are set, as shown on Figure 6-right, as a function of $m_{H}$. This analysis excludes a SM-like Higgs boson in the $275-600 \mathrm{GeV}$ range at 95\% CL.
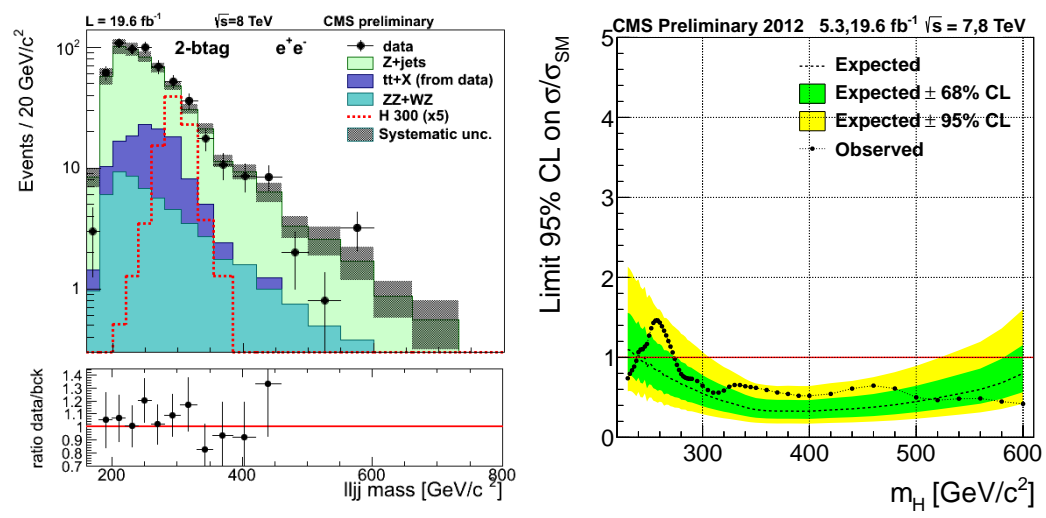

Figure 6: Left: $m_{\ell \ell j j}$ distribution after final selection for events with 2 electrons and $2 b$-tagged jets. Right: Observed and expected $95 \% \mathrm{CL}$ upper limit on $\sigma / \sigma_{\mathrm{SM}}$. Figures from Ref. [17].

\section{Summary and conclusions}

Natural extensions of the SM Higgs sector predict heavy SM-like resonances, and are possible scenarios under the current measurements of the newly discovered boson with $125 \mathrm{GeV}$ mass. $H \rightarrow$ $W W$ and $H \rightarrow Z Z$ are the preferred decays to explore the high mass regime, and both ATLAS and CMS have performed searches in these channels. No significant excesses have been observed and large high-mass ranges have been excluded by both experiments, assuming SM-like scenarios, as well as under the BSM heavy EW singlet model, that currently favours a narrow-width resonance.

\section{References}

[1] ATLAS Collaboration, JINST 3 (2008) S08003.

[2] CMS Collaboration, JINST 3 (2008) S08004.

[3] ATLAS Collaboration, Phys. Lett. B 716 (2012) 1 [hep-ex/1207. 7214].

[4] CMS Collaboration, Phys. Lett. B 716 (2012) 30 [hep-ex/1207. 7235].

[5] ATLAS Collaboration, Phys. Lett. B 726 (2013) 88 [hep-ex/1307.1427].

[6] CMS Collaboration, CMS-PAS-HIG-13-005 [cds . cern. ch/record/1542387].

[7] LHC Higgs Cross Section Working Group, Heinemeyer, S., Mariotti, C., Passarino, G. and Tanaka, R. (Eds.) [hep-ph/1307.1347].

[8] ATLAS Collaboration, Phys. Lett. B 718 (2012) 391 [hep-ex/1206. 6074].

[9] CMS Collaboration, CMS PAS HIG-13-008 [cds . cern. ch/record/1546778].

[10] ATLAS Collaboration, ATLAS-CONF-2013-067 [cds.cern.ch/record/1562879].

[11] CMS Collaboration, CMS PAS HIG-13-003 [cds . cern. ch/record/1523673].

[12] ATLAS Collaboration, ATLAS-CONF-2013-013 [cds.cern.ch/record/1523699].

[13] CMS Collaboration, CMS PAS HIG-13-002 [cds . cern. ch/record/1523767].

[14] ATLAS Collaboration, Phys. Lett. B 717 (2012) 29 [hep-ex/1205.6744].

[15] CMS Collaboration, CMS-PAS-HIG-13-014 [cds . cern. ch/record/1546776].

[16] ATLAS Collaboration, Phys. Lett. B 717 (2012) 70 [hep-ex/1206.2443].

[17] CMS Collaboration, CMS-PAS-HIG-12-024 [cds . cern. ch/record/1564157]. 